\title{
Stillbirth rate in singleton pregnancies: a 20-year retrospective study from a public obstetric unit in Hong Kong
}

\author{
Sani TK Wong, WT Tse, SL Lau, Daljit S Sahota, TY Leung *
}

This article was published on 21 Jan 2022 at www.hkmj.org.

\section{A B S T R A C T}

Introduction: Although the stillbirth rate is low in Hong Kong, up to $50 \%$ of stillbirths have unclassifiable causes and up to one third of stillbirths have unexplained causes. This retrospective study investigated the underlying causes of singleton stillbirths in Hong Kong.

Methods: This study examined the prevalences and causes of stillbirths in a university tertiary obstetric unit between 2000 and 2019. Medical records were reviewed for all singleton pregnancies complicated by stillbirths. Causes of stillbirth were determined via clinical assessments and laboratory findings, then compared between 2000-09 and 2010-19.

Results: Overall perinatal mortality significantly decreased by $16.7 \%$, from $5.50 / 1000$ in $2000-09$ to $4.59 / 1000$ in 2010-19; the singleton stillbirth rate slightly decreased (from 3.27/1000 to 2.91/1000). These changes were related to early prenatal diagnostic improvements concerning congenital malformations and genetic disorders. Pre-eclampsia prevalence among singleton pregnancies increased from $1.5 \%$ to $1.7 \%$ because of increasing maternal age; the stillbirth rate among patients with preeclampsia decreased from $2.5 \%$ to $1.4 \%$. Fetal growth restriction of unknown cause contributed to $16 \%$ of all stillbirths; this prevalence did not change over time. Moreover, fetal growth restriction was not diagnosed during routine antenatal care in $43.5 \%$ of patients. Thirty-six percent of all stillbirths were unexplained. The prevalences of stillbirth associated with chorioamnionitis and placental abruption did not change over time.

Conclusions: Causes of stillbirth in Hong Kong have changed in the past 20 years because of altered demographic characteristics and improved prenatal testing. Further improvements should focus on early fetal growth restriction detection and pre-eclampsia prevention.

\section{Hong Kong Med J 2022;28:285-93

https://doi.org/10.12809/hkmj219361

\section{STK Wong, MB, ChB}

WT Tse, MB, ChB, MRCOG

SL Lau, MB, ChB, MRCOG

DS Sahota, $\mathrm{PhD}$

TY Leung *, MD, FRCOG

Department of Obstetrics and Gynaecology, The Chinese University of Hong Kong, Hong Kong

* Corresponding author: tyleung@cuhk.edu.hk

New knowledge added by this study

- The stillbirth rate among singleton pregnancies in Hong Kong is declining (from 3.27/1000 in 2000-09 to 2.91/1000 in 2010-19), mainly because of reductions in congenital malformations and genetic diseases.

- Pre-eclampsia is becoming more prevalent (from 1.5\% in 2000-09 to 1.7\% in 2010-19), although the preeclampsia-related stillbirth rate has decreased (from $2.5 \%$ to $1.4 \%$ ).

- Fetal growth restriction (FGR) remains a common cause of stillbirths ( $16 \%$ of all stillbirths), and $43.5 \%$ of FGRrelated stillbirths were undiagnosed during routine antenatal care.

Implications for clinical practice or policy

- Primary prevention of pre-eclampsia through first trimester screening and aspirin prophylaxis is essential for improving maternal and fetal health.

- Antenatal detection of FGR should be improved using more accurate sonographic and biochemical tests.

- Territory-wide perinatal mortality monitoring is important for maintaining the standard of perinatal care in Hong Kong.

\section{Introduction}

Compared with many other regions worldwide, Hong Kong has one of the lowest perinatal mortality rates, defined as the total number of stillbirths and early neonatal deaths per 1000 births. ${ }^{1}$ Worldwide, there are consistent definitions of early and late neonatal deaths (ie, the death of a livebirth in the first 7 days and 28 days after birth, respectively); however, the definition of stillbirth varies among regions. Hong Kong has been using the United Kingdom's definition of stillbirth, which is 'a baby delivered with no signs of life at or after 24 weeks of gestation.' ${ }^{1}$ In 


\section{單胎妊娠死胎率：香港公立醫院產科20年回顧 性研究 \\ 王子琦、謝穎婷、劉素玲、DS Sahota、梁德楊}

引言：雖然香港的死胎率偏低, 但高達 $50 \%$ 的死胎原因不能分類, 以 及高達三分之一的死胎原因不明。這項回顧性研究檢視香港單胎死胎 的根本原因。

方法：本研究檢視2000年至2019年期間香港一所教學醫院產科的死胎 現患率和原因, 並審閲所有單胎妊娠併發死胎的醫療記錄。透過臨床 評估和化驗結果確定死胎原因, 然後把2000-09和2010-19的數據進行 比較。

結果：總體圍產期死亡率從2000-09年的5.50/1000，顯著下降 16.7\% 至2010-19年的4.59/1000；單胎死產率略有下降（從3.27/1000降至 $2.91 / 1000 ） 。$ 這與先天畸形和遺傳疾病的早期產前診斷有所改善相 關。由於產婦年齡上升, 單胎妊娠毒血症的現患率由 $1.5 \%$ 增至 $1.7 \%$; 而毒血症患者的死胎率則從 $2.5 \%$ 下降到 $1.4 \%$ 。原因不明的胎兒生長 受限（FGR）佔所有死胎 $16 \%$, 其現患率並沒有隨時間而改變。此 外, $43.5 \%$ 患者未能在常規產前檢查時診斷出FGR。36\%死胎原因不 明。與絨毛膜羊膜炎和胎盤早剝相關的死胎現患率並未隨時間而改 變。

結論：由於人口特徵的改變和產前檢測有所改善, 香港死胎的原因在 過去 20 年發生了變化。應側重進一步改善於早期FGR檢測和預防毒血 症。 different coding systems that are used by obstetric units in Hong Kong.

This study was performed to review the overall perinatal mortality in a tertiary centre in Hong Kong, and specifically explored the causes, the associated risk factors and trends of stillbirth in singleton pregnancies. Neonatal deaths and perinatal mortalities in multiple pregnancies will be reported separately.

\section{Methods}

\section{Study setting}

This study retrospectively reviewed data collected from 1 January 2000 to 31 December 2019, in the Prince of Wales Hospital in Hong Kong. The hospital serves a population of approximately 1.7 million in the New Territories East region of Hong Kong, with an annual delivery rate of approximately 6000 to 7000 births. The obstetric unit is also a tertiary centre that receives complicated maternal and fetal cases referred from other hospitals as well as a maternal fetal medicine training centre accredited by both The Royal College of Obstetricians and Gynaecologists (https://www.rcog.org.uk) and The Hong Kong College of Obstetricians and Gynaecologists (www.hkcog.org.hk). The standard antenatal and obstetric care, as well as the investigation of stillbirth and neonatal death are described in the Supplementary Appendix.

contrast, some countries use 20 weeks, 22 weeks, or $500 \mathrm{~g}$ as a threshold for stillbirth. ${ }^{2}$ To facilitate global comparisons, the World Health Organization has stratified stillbirth into early (death at a birthweight $>500 \mathrm{~g}$ or at a gestational age $\geq 22$ weeks) and late fetal death (death at a birthweight $\geq 1000 \mathrm{~g}$ or at a gestational age $\geq 28$ weeks). ${ }^{3}$

The estimated global stillbirth rate was 18.4/1000 births in 2015. ${ }^{4}$ Estimated stillbirth rates are significantly lower in developed countries (approximately 3.4/1000 births); the highest stillbirth rate has been reported in sub-Saharan Africa regions (28.7/1000 births). ${ }^{4}$ Intrapartum stillbirths comprise up to $57 \%$ of all reported stillbirths in South Asia; most are related to obstetric emergencies. ${ }^{5}$ Thus, the stillbirth rate is a potential indicator of a country's healthcare system quality; variations in intrapartum stillbirth rates among countries may reflect the readiness of health facilities to provide adequate intrapartum care and ensure that trained birth attendants are available for delivery. ${ }^{5}$ Therefore, the United Nations has included stillbirth prevention as a major Sustainable Development Goal. ${ }^{6}$

The stillbirth rate is consistently low in Hong Kong (2/1000 births during the period from 2004 to 2014). ${ }^{1}$ However, up to $50 \%$ of stillbirths have unclassifiable causes and up to one third of stillbirths have unexplained causes, which may reflect inconsistencies and limitations regarding the

\section{Data collection and analysis}

Data of all deliveries including maternal demographic data (eg, ethnicity, maternal age, height, body weight, body mass index [BMI], underlying medical diseases, and obstetric history), obstetric and perinatal outcomes were retrieved from a hospital-specific Obstetric Specialty Clinical Information System that is used to record maternal and perinatal outcomes after birth. ${ }^{7}$ Stillbirths (defined as fetal death that occurred at or after 24 weeks; late stillbirth occurred at or after 28 weeks $)^{3}$ were identified from the database and their details were further retrieved from hospital electronic records. All stillbirths were included regardless of their booking status or whether their deaths were occurred before admission to our unit; however, the booking status was incorporated into the analysis.

The stillbirth rate was calculated as the number of stillbirths divided by the total number of births (after 24 weeks). The stillbirth rates of singleton pregnancies were compared between 2000-09 and 2010-19.

\section{Statistical analysis}

Continuous variables were compared by the independent samples $t$ test or Mann-Whitney $U$ test for parametric and non-parametric data, 
respectively. Categorical variables were compared by the Chi squared test or Fisher's exact test, as appropriate. The level of significance was set at a two-sided $\mathrm{P}$ value of $<0.05$. Data analysis was performed using SPSS (Windows version 22.0; IBM Corp, Armonk [NY], United States).

\section{Results}

\section{Mortality during the study period}

During the 20-year study period, there were 128967 babies delivered. Among them were 429 stillbirths, 159 early neonatal deaths, and 59 late neonatal deaths. The total mortality rate was 5.02 per 1000 births and the perinatal mortality rate was 4.56 per 1000 births. The total mortality rate was significantly lower during 2010-19 than during 2000-09 (4.59 per 1000 births vs 5.50 per 1000 births; $\mathrm{P}=0.023$ ). The perinatal mortality rate was also significantly lower in 2010-19 (4.18 per 1000 births vs 5.00 per 1000 births; $\mathrm{P}=0.035)$, although the rates of stillbirths and late stillbirths were not significantly different between 2000-09 and 2010-19 (Table 1).

Table 2 shows all mortality data for singleton pregnancies. Among 124665 singleton babies, the respective total mortality, perinatal mortality and stillbirth rates were 4.56/1000, 4.17/1000 and $3.08 / 1000$. Among the 384 singleton stillbirths,
$95(24.7 \%)$ occurred between 24 and 27 weeks, while $289(75.3 \%)$ occurred thereafter; thus, the late stillbirth rate was $2.33 / 1000$. The detailed distribution of gestational ages among stillbirths is shown in Table 3. There were $23(6 \%)$ cases of intrapartum death $(0.18 / 1000$ births $)$; these were caused by placental abruption $(n=11)$, known lethal fetal anomalies $(n=7)$, chorioamnionitis $(n=2)$, uterine rupture $(\mathrm{n}=1)$, maternal diabetic ketoacidosis $(\mathrm{n}=1)$, and umbilical cord accident-cord ulceration related to duodenal atresia $(n=1){ }^{8}$

\section{Singleton stillbirths: causes and potential risk factors}

Table 4 shows the direct causes of the 384 singleton stillbirths that occurred during the study period. The most common leading cause was fetal growth restriction (FGR) $[\mathrm{n}=61,15.9 \%$ of singleton stillbirths]; 41 of these 61 cases (67.2\%) were not diagnosed before birth. The next most common leading causes were chorioamnionitis $(n=43,11.2 \%$ of singleton stillbirths), congenital malformations and genetic abnormalities ( $n=35,9.1 \%$ of singleton stillbirths), placental abruption ( $n=31,8.1 \%$ of singleton stillbirths), and pre-eclampsia $(n=24,6.3 \%$ of singleton stillbirths). Pre-eclampsia also occurred in 11 cases of placental abruption, one case of genetic

TABLE I. Comparison of stillbirth rates, neonatal death (NND) rates, and total and perinatal mortality rates (per I000 births) among both singleton and multiple pregnancies between 2000-09 and 2010-19*

\begin{tabular}{|c|c|c|c|c|}
\hline & Total, No. (/1000) & $2000-09$, No. $(/ 1000)$ & 2010-19, No. (/1000) & $P$ value \\
\hline All births & 128967 & 60332 & 68635 & \\
\hline Total mortality & $647(5.02)$ & $332(5.50)$ & $315(4.59)$ & 0.023 \\
\hline Perinatal mortality & $588(4.56)$ & $301(5.00)$ & $287(4.18)$ & 0.035 \\
\hline Total stillbirths & 429 (3.33) & $217(3.60)$ & 212 (3.09) & 0.125 \\
\hline All births $\geq 28$ weeks & 128447 & 60091 & 68356 & \\
\hline Late stillbirth & $313(2.44)$ & $153(2.55)$ & $160(2.34)$ & 0.491 \\
\hline
\end{tabular}

Total mortality = all stillbirths and NND; perinatal mortality = all stillbirths and early NND; late stillbirth = stillbirth at or after 28 gestational weeks

TABLE 2. Comparison of stillbirth rates, neonatal death (NND) rates, and total and perinatal mortality rates (per I000 births) among singleton pregnancies between 2000-09 and 2010-19*

\begin{tabular}{|c|c|c|c|c|}
\hline & Total, No. (/1000) & $2000-09$, No. $(/ 1000)$ & 2010-19, No. (/1000) & $P$ value \\
\hline All births & 124665 & 58634 & 66031 & \\
\hline Total mortality & $568(4.56)$ & $289(4.93)$ & $279(4.23)$ & 0.072 \\
\hline Perinatal mortality & $520(4.17)$ & $266(4.54)$ & $254(3.85)$ & 0.065 \\
\hline Total stillbirths & $384(3.08)$ & 192 (3.27) & $192(2.91)$ & 0.265 \\
\hline All births $\geq 28$ weeks & 124242 & 58434 & 65808 & \\
\hline Late stillbirth & $289(2.33)$ & $140(2.40)$ & $149(2.26)$ & 0.673 \\
\hline
\end{tabular}

* Total mortality = all stillbirths and NND; perinatal mortality = all stillbirths and early NND; late stillbirth = stillbirth at or after 28 gestational weeks 
abnormality (Haemoglobin Barts), and one case of chorioamnionitis; importantly, pre-eclampsia was not regarded as the leading cause in these 13 cases. There were 139 unexplained stillbirths and comprised about one third of all singleton stillbirths.

Table 5 shows the maternal characteristics for all stillbirths and for the subgroups of unexplained stillbirths and FGR-related stillbirths in singleton pregnancies. Compared with mothers in the livebirth group (excluding neonatal death), mothers in the stillbirth group were significantly older ( $30.7 \pm 5.5$ years vs $31.8 \pm 5.8$ years; $\mathrm{P}<0.001$ ); greater proportions of mothers in the stillbirth group had advanced maternal age $\geq 35$ years $(24.1 \%$ vs $33.3 \%$; $\mathrm{P}<0.001)$ and $\geq 40$ years $(3.9 \%$ vs $6.8 \% ; \mathrm{P}=0.004)$. Mothers in the stillbirth group also had higher BMI

TABLE 3. Distribution of gestational ages when stillbirths occurred or were diagnosed $^{*}$

\begin{tabular}{lcccc}
\hline Gestation, wk & Total $(\mathbf{n = 3 8 4})$ & $\mathbf{2 0 0 0 - 0 9}(\mathbf{n = 1 9 2})$ & $\mathbf{2 0 1 0 - 1 9}(\mathbf{n = 1 9 2})$ & P value \\
\hline $24-27$ & $95(24.7 \%)$ & $52(27.1 \%)$ & $43(22.4 \%)$ & 0.470 \\
$28-31$ & $84(21.9 \%)$ & $37(19.3 \%)$ & $47(24.5 \%)$ & \\
$32-36$ & $90(23.4 \%)$ & $43(22.4 \%)$ & $47(24.5 \%)$ & \\
$37-38$ & $63(16.4 \%)$ & $29(15.1 \%)$ & $34(17.7 \%)$ & \\
$39-40$ & $44(11.5 \%)$ & $26(13.5 \%)$ & $18(9.4 \%)$ & \\
$\geq 41$ & $8(2.1 \%)$ & $5(2.6 \%)$ & $3(1.6 \%)$ & \\
\hline
\end{tabular}

Data are shown as No. (\%), unless otherwise specified at booking $\left(22.8 \pm 3.6 \mathrm{~kg} / \mathrm{m}^{2}\right.$ vs $23.4 \pm 3.9 \mathrm{~kg} / \mathrm{m}^{2}$; $\mathrm{P}=0.003$ ); greater proportions of mothers in the stillbirth group had BMI $\geq 30 \mathrm{~kg} / \mathrm{m}^{2}(3.7 \%$ vs $6.3 \%$; $\mathrm{P}=0.012)$. The prevalences of nulliparity $(52.2 \%$ vs $57.7 \% ; \mathrm{P}=0.027)$, non-booked status $(8.0 \%$ vs $22.9 \%$, $\mathrm{P}<0.001)$, and non-Chinese Asian ethnicity $(2.6 \%$ vs $5 \% ; \mathrm{P}=0.018)$ were also significantly higher in the stillbirth group. In the unexplained stillbirth subgroup, only nulliparity and non-booked status remained significant risk factors. In the FGR-related stillbirth subgroup, the mothers were more likely to be non-Chinese, non-booked cases, nulliparous, older, and obese (BMI $\left.\geq 30 \mathrm{~kg} / \mathrm{m}^{2}\right)$.

Compared with the livebirth group, the prevalences of several medical diseases were also higher in the stillbirth group, including pre-eclampsia ( $1.6 \%$ vs $9.6 \% ; \mathrm{P}<0.001)$, liver diseases $(0.1 \%$ vs $0.8 \%$; $\mathrm{P}<0.001)$, and immunological diseases $(0.2 \%$ vs $0.8 \%$; $\mathrm{P}=0.033$ ). However, histories of other pre-existing medical condition such as diabetes and chronic hypertension were not associated with stillbirth. There was a lower prevalence of gestational diabetes (8.2\% vs $4.2 \% ; \mathrm{P}=0.004)$ in the stillbirth group.

\section{Changes in stillbirth causes and risk factors over time}

Comparing 2000-09 and 2010-19, there was a trend towards reduction in stillbirth rate $(3.27 / 1000$ vs 2.91/1000; $\mathrm{P}=0.265$ ), although this difference was not statistically significant. Among the direct causes of stillbirths, there was a significant reduction in the prevalence of congenital malformations and

TABLE 4. Causes of stillbirths among singleton pregnancies listed according to the order of their prevalence, compared between 2000-09 and 2010-19

\begin{tabular}{|c|c|c|c|c|}
\hline Causes & $\begin{array}{l}\text { Total, } n=124665 \\
\text { No. }(/ 1000)\end{array}$ & $\begin{array}{c}2000-09, n=58634 \\
\text { No. }(/ 1000)\end{array}$ & $\begin{array}{c}2010-19, \mathrm{n}=66031 \\
\text { No. }(/ 1000)\end{array}$ & $P$ value \\
\hline Total stillbirths & $384(3.08)$ & $192(3.27)$ & $192(2.91)$ & 0.265 \\
\hline Fetal growth restriction & $61(0.49)$ & $29(0.49)$ & $32(0.48)$ & 0.961 \\
\hline Chorioamnionitis* $^{*}$ & $43(0.34)$ & $23(0.39)$ & $20(0.30)$ & 0.487 \\
\hline $\begin{array}{l}\text { Congenital malformations and } \\
\text { genetic abnormalities }{ }^{*}\end{array}$ & $35(0.28)$ & $26(0.44)$ & $9(0.14)$ & 0.002 \\
\hline Placental abruption $^{\dagger}$ & $31(0.25)$ & $16(0.27)$ & $15(0.23)$ & 0.741 \\
\hline Pre-eclampsia ${ }^{\ddagger}$ & $24(0.19)$ & $15(0.26)$ & $9(0.14)$ & 0.189 \\
\hline Other placental pathologies & $23(0.18)$ & $6(0.10)$ & $17(0.26)$ & 0.071 \\
\hline $\begin{array}{l}\text { Other maternal or surgical } \\
\text { complications of pregnancy }\end{array}$ & $14(0.11)$ & $6(0.10)$ & $8(0.12)$ & 0.796 \\
\hline Cord-related pathology/accident & $11(0.09)$ & $6(0.10)$ & $5(0.08)$ & 0.765 \\
\hline Fetal-placental haemorrhage & $2(0.02)$ & 0 & $2(0.03)$ & 0.502 \\
\hline Uterine rupture & $1(0.01)$ & 0 & $1(0.02)$ & 1.000 \\
\hline Unexplained & $139(1.12)$ & 64 (1.09) & $75(1.14)$ & 0.882 \\
\hline
\end{tabular}

* One case of chorioamnionitis (2010-19) and one case of Haemoglobin Barts disease also involved pre-eclampsia (2000-09)

Eleven placental abruption-related stillbirths also involved pre-eclampsia (6 and 5 cases in 2000-09 and 2010-19, respectively)

Eight pre-eclampsia-related stillbirths involved birthweight below the first centile 
TABLE 5. Comparisons of maternal demographic characteristics between livebirths and all stillbirths, and between unexplained stillbirths and fetal growth restriction-related stillbirths, for all singleton pregnancies from 2000 to $2019^{*}$

\begin{tabular}{|c|c|c|c|c|c|c|c|}
\hline & $\begin{array}{c}\text { Livebirth } \\
\text { (excluding NND), } \\
n=124097\end{array}$ & $\begin{array}{c}\text { All stillbirth, } \\
n=384\end{array}$ & $P$ value & $\begin{array}{l}\text { Unexplained } \\
\text { stillbirth, } n=139\end{array}$ & $P$ value & $\begin{array}{c}\text { FGR-related } \\
\text { stillbirth, } n=61\end{array}$ & $P$ value \\
\hline Ethnicity & & & 0.018 & & 0.962 & & 0.022 \\
\hline Chinese & 120843 (97.4\%) & 365 (95.1\%) & & $136(97.8 \%)$ & & 56 (91.8\%) & \\
\hline Other Asian & $2740(2.2 \%)$ & $16(4.2 \%)$ & & $3(2.2 \%)$ & & $4(6.6 \%)$ & \\
\hline Caucasians & $514(0.4 \%)$ & $3(0.8 \%)$ & & 0 & & $1(1.6 \%)$ & \\
\hline Non-booked case & $9916(8.0 \%)$ & $88(22.9 \%)$ & $<0.001$ & $23(16.5 \%)$ & 0.001 & 12 (19.7\%) & 0.001 \\
\hline Parity & & & 0.027 & & 0.038 & & 0.019 \\
\hline Nulliparous & 64789 (52.2\%) & 221 (57.9\%) & & 87 (62.6\%) & & $41(67.2 \%)$ & \\
\hline Multiparous & 59311 (47.8\%) & $161(42.1 \%)$ & & $52(37.4 \%)$ & & $20(32.8 \%)$ & \\
\hline \multicolumn{8}{|l|}{ Maternal age, y } \\
\hline Mean \pm SD & $30.7 \pm 5.5$ & $31.8 \pm 5.8$ & $<0.001$ & $30.9 \pm 5.6$ & 0.672 & $32.8 \pm 5.8$ & 0.003 \\
\hline$\leq 19$ & $2206(1.8 \%)$ & $7(1.8 \%)$ & 0.655 & $3(2.2 \%)$ & 0.883 & 0 & 0.630 \\
\hline$\geq 35$ & 29854 (24.1\%) & $128(33.3 \%)$ & $<0.001$ & $38(27.3 \%)$ & 0.541 & $26(42.6 \%)$ & 0.001 \\
\hline$\geq 40$ & $4893(3.9 \%)$ & $26(6.8 \%)$ & 0.004 & 7 (5.0\%) & 0.777 & $8(13.1 \%)$ & $<0.001$ \\
\hline \multicolumn{8}{|l|}{ Booking BMI, kg/m² } \\
\hline Mean \pm SD & $22.8 \pm 3.6$ & $23.4 \pm 3.9$ & 0.003 & $22.8 \pm 3.4$ & 0.986 & $23.7 \pm 4.4$ & 0.051 \\
\hline$<18.5$ & $9084(7.3 \%)$ & $22(5.7 \%)$ & 0.277 & $8(5.8 \%)$ & 0.586 & $4(6.6 \%)$ & 0.986 \\
\hline$\geq 25$ & 27747 (22.4\%) & $93(24.2 \%)$ & 0.417 & 27 (19.4\%) & 0.788 & $14(23.0 \%)$ & 0.966 \\
\hline$\geq 30$ & $4591(3.7 \%)$ & $24(6.3 \%)$ & 0.012 & $4(2.9 \%)$ & 0.916 & $8(13.1 \%)$ & $<0.001$ \\
\hline \multicolumn{8}{|l|}{ Diabetes-related disorders } \\
\hline Pre-existing diabetes & $380(0.3 \%)$ & $3(0.8 \%)$ & 0.093 & NA & & 0 & NA \\
\hline Gestational diabetes & $10157(8.2 \%)$ & $16(4.2 \%)$ & 0.004 & & & $1(1.6 \%)$ & 0.061 \\
\hline \multicolumn{8}{|l|}{ Hypertension-related disorders } \\
\hline Chronic hypertension (only) & $354(0.3 \%)$ & $1(0.3 \%)$ & 0.698 & NA & & 0 & NA \\
\hline Pre-eclampsia & $1927(1.6 \%)$ & $37(9.6 \%)$ & $<0.001$ & & & 0 & \\
\hline Gestational hypertension & $1383(1.1 \%)$ & 0 & 0.066 & & & 0 & \\
\hline \multicolumn{8}{|l|}{ Other underlying medical disease } \\
\hline Cardiac & $1397(1.1 \%)$ & $1(0.3 \%)$ & 0.108 & NA & & $1(1.6 \%)$ & 0.704 \\
\hline Renal & $615(0.5 \%)$ & $3(0.8 \%)$ & 0.426 & & & $1(1.6 \%)$ & 0.204 \\
\hline Liver & $124(0.1 \%)$ & $3(0.8 \%)$ & $<0.001$ & NA & & 0 & NA \\
\hline Respiratory & $2294(1.8 \%)$ & $7(1.8 \%)$ & 0.970 & NA & & 0 & NA \\
\hline Gastrointestinal & $160(0.1 \%)$ & 0 & 0.481 & NA & & 0 & NA \\
\hline Neurological & $328(0.3 \%)$ & 0 & 0.313 & NA & & 0 & NA \\
\hline Psychiatric & $1267(1.0 \%)$ & $3(0.8 \%)$ & 0.641 & NA & & 0 & NA \\
\hline Immunological & $301(0.2 \%)$ & $3(0.8 \%)$ & 0.033 & NA & & 0 & NA \\
\hline Thyroid & 2617 (2.1\%) & $9(2.3 \%)$ & 0.769 & NA & & 0 & NA \\
\hline
\end{tabular}

Abbreviations: $\mathrm{BMI}=$ body mass index; FGR = fetal growth restriction; NA = not applicable; NND = neonatal death; $\mathrm{SD}=$ standard deviation

* Data are shown as No. (\%), unless otherwise specified

genetic abnormalities $(0.44 / 1000$ vs $0.14 / 1000$; significance $(0.10 / 1000$ vs $0.26 / 1000 ; \mathrm{P}=0.071)$. The $\mathrm{P}=0.002)$. There was a trend towards reduction prevalences of FGR, chorioamnionitis, placental in pre-eclampsia-related stillbirths $(0.26 / 1000$ vs abruption, other maternal or surgical complications $0.14 / 1000 ; \mathrm{P}=0.189$ ), although this difference was of pregnancy, cord-related pathology/accident, not statistically significant. There was an increasing fetal-placental haemorrhage, uterine rupture, and trend of singleton stillbirths related to other placental unexplained stillbirth did not change over time pathologies although it did not reach statistical (Table 4). 
TABLE 6. Comparison of maternal characteristics and mode of delivery between 2000-09 and 2010-19 for all singleton pregnancies*

\begin{tabular}{|c|c|c|c|}
\hline & $\begin{array}{l}2000-09 \\
n=58634\end{array}$ & $\begin{array}{l}2010-19 \\
n=66031\end{array}$ & $P$ value \\
\hline Ethnicity & & & $<0.001$ \\
\hline Chinese & $56973(97.2 \%)$ & 64409 (97.5\%) & \\
\hline Other Asian & $1434(2.4 \%)$ & $1331(2.0 \%)$ & \\
\hline Caucasians & $227(0.4 \%)$ & $291(0.5 \%)$ & \\
\hline \multicolumn{4}{|l|}{ Maternal age, y } \\
\hline Mean $\pm S D$ & $29.9 \pm 5.3$ & $31.4 \pm 5.6$ & $<0.001$ \\
\hline$\leq 19$ & $1112(1.9 \%)$ & 1107 (1.7\%) & 0.004 \\
\hline$\geq 35$ & $11834(20.2 \%)$ & 18207 (27.6\%) & $<0.001$ \\
\hline$\geq 40$ & $1859(3.2 \%)$ & $3071(4.7 \%)$ & $<0.001$ \\
\hline \multicolumn{4}{|l|}{$\mathrm{BMI}$ at booking, $\mathrm{kg} / \mathrm{m}^{2}$} \\
\hline Mean \pm SD & $22.9 \pm 3.6$ & $22.6 \pm 3.6$ & $<0.001$ \\
\hline$<18.5$ & $3918(6.7 \%)$ & 5197 (7.9\%) & $<0.001$ \\
\hline$\geq 25$ & $14045(24.0 \%)$ & $13839(21.0 \%)$ & $<0.001$ \\
\hline$\geq 30.0$ & $2087(3.6 \%)$ & $2535(3.8 \%)$ & 0.010 \\
\hline Parity & & & 0.065 \\
\hline Nulliparous & $30786(52.5 \%)$ & $34326(52.0 \%)$ & \\
\hline Multiparous & $27848(47.5 \%)$ & $31705(48.0 \%)$ & \\
\hline Non-booked case & $7976(13.6 \%)$ & 2067 (3.1\%) & $<0.001$ \\
\hline \multicolumn{4}{|l|}{ Diabetes-related disorders } \\
\hline Pre-existing diabetes & $148(0.3 \%)$ & $235(0.4 \%)$ & 0.001 \\
\hline Gestational diabetes & $3956(6.7 \%)$ & $6224(9.4 \%)$ & $<0.001$ \\
\hline \multicolumn{4}{|l|}{ Hypertension-related disorders } \\
\hline Chronic hypertension (only) & $61(0.1 \%)$ & $294(0.4 \%)$ & $<0.001$ \\
\hline Pre-eclampsia & $888(1.5 \%)$ & $1090(1.7 \%)$ & 0.058 \\
\hline Gestational hypertension & $224(0.4 \%)$ & $1160(1.8 \%)$ & $<0.001$ \\
\hline \multicolumn{4}{|l|}{ Other underlying medical disease } \\
\hline Cardiac & $712(1.2 \%)$ & $690(1.0 \%)$ & 0.005 \\
\hline Renal & $370(0.6 \%)$ & $254(0.4 \%)$ & $<0.001$ \\
\hline Liver & $43(0.1 \%)$ & $87(0.1 \%)$ & 0.002 \\
\hline Respiratory & $880(1.5 \%)$ & $1425(2.2 \%)$ & $<0.001$ \\
\hline Gastrointestinal & $129(0.2 \%)$ & $32(0.1 \%)$ & $<0.001$ \\
\hline Neurological & $151(0.3 \%)$ & $177(0.3 \%)$ & 0.717 \\
\hline Psychiatric & $394(0.7 \%)$ & $877(1.3 \%)$ & $<0.001$ \\
\hline Immunological & $110(0.2 \%)$ & $197(0.3 \%)$ & $<0.001$ \\
\hline Thyroid & $1125(1.9 \%)$ & $1494(2.3 \%)$ & $<0.001$ \\
\hline Induction of labour & $10293(17.6 \%)$ & $17087(25.9 \%)$ & $<0.001$ \\
\hline \multicolumn{4}{|l|}{ Mode of delivery } \\
\hline NSD & $39592(67.5 \%)$ & $45418(68.8 \%)$ & $<0.001$ \\
\hline Instrumental & $6449(11.0 \%)$ & $5058(7.7 \%)$ & \\
\hline Vaginal breech & $156(0.3 \%)$ & $208(0.3 \%)$ & \\
\hline Elective CS & 3713 (6.3\%) & 7091 (10.7\%) & \\
\hline Emergency CS & 8724 (14.9\%) & $8256(12.5 \%)$ & \\
\hline All vaginal & 46197 (78.8\%) & $50684(76.8 \%)$ & $<0.001$ \\
\hline All CS & $12437(21.2 \%)$ & $15347(23.2 \%)$ & \\
\hline
\end{tabular}

Abbreviations: $\mathrm{BMI}=$ body mass index; $\mathrm{CS}=$ caesarean section; $\mathrm{NSD}=$ normal spontaneous delivery; SD = standard deviation

Data are shown as No. (\%), unless otherwise specified
Between 2000-09 and 2010-19, there were several significant changes in maternal demographics (Table 6), including a higher maternal age over time $(29.9 \pm 5.3$ years vs $31.4 \pm 5.6$ years; $\mathrm{P}<0.001)$; in 2010-19, a greater proportion of mothers had advanced maternal age $\geq 35$ years $(20.2 \%$ vs $27.6 \%$; $\mathrm{P}<0.001)$ and $\geq 40$ years $(3.2 \%$ vs $4.7 \% ; \mathrm{P}<0.001)$. The mean booking BMI was significantly reduced in $2010-19\left(22.9 \pm 3.6 \mathrm{~kg} / \mathrm{m}^{2}\right.$ vs $22.6 \pm 3.6 \mathrm{~kg} / \mathrm{m}^{2}$; $\mathrm{P}<0.001)$, with greater prevalence of underweight (BMI at booking $<18.5 \mathrm{~kg} / \mathrm{m}^{2} ; 6.7 \%$ vs $7.9 \% ; \mathrm{P}<0.001$ ), although a greater prevalence of obesity was also observed (BMI at booking $>30 \mathrm{~kg} / \mathrm{m}^{2} ; 3.6 \%$ vs $3.8 \%$, $\mathrm{P}=0.01$ ) [Table 6]. The prevalence of non-booked cases was also significantly reduced ( $13.6 \%$ vs $3.1 \%$; $\mathrm{P}<0.001)$. Chinese ethnicity remained dominant (97.2\% vs $97.5 \% ; \mathrm{P}<0.001)$ and the proportions of nulliparous mothers were similar $(52.5 \%$ vs $52.0 \%$; $\mathrm{P}=0.065)$. In 2010-19, there were higher prevalence of pre-existing diabetes $(0.3 \%$ vs $0.4 \% ; \mathrm{P}=0.001)$, gestational diabetes $(6.7 \%$ vs $9.4 \% ; \quad \mathrm{P}<0.001)$, chronic hypertension $(0.1 \%$ vs $0.4 \% ; \mathrm{P}<0.001)$ and gestational hypertension ( $0.4 \%$ vs $1.8 \%$; $\mathrm{P}<0.001)$; the caesarean delivery rate also increased $(21.2 \%$ vs $23.2 \%$; $<0.001)$. Other changes in the prevalences of medical diseases are summarised in Table 6.

\section{Discussion}

\section{Changes in perinatal mortality}

This investigation of perinatal mortality in Hong Kong showed that the overall perinatal mortality rate was significantly reduced by $16.7 \%$ from $5.50 / 1000$ in $2000-09$ to $4.59 / 1000$ in $2010-19$. This is a combined effect of reductions in stillbirths and neonatal deaths, although these individual trends (ie, rates of stillbirth and neonatal death) were not significantly different over time. Compared with the stillbirth rate of 4.44/1000 reported by our unit in $1994,{ }^{9}$ we observed gradual declines to $3.60 / 1000$ in 2000-09 and 3.09/1000 in 2010-19. Because our hospital is a tertiary referral centre, it is reasonable that our stillbirth rate is slightly higher than the rate determined in a territory-wide audit performed by the Hong Kong College of Obstetricians and Gynaecologists (2/1000 births). ${ }^{1}$

The intrapartum death rate in Hong Kong was considerably reduced from $0.96 / 1000$ (22\% of all stillbirths) in the $1990 \mathrm{~s}^{9}$ to $0.2 / 1000$ in the present study; intrapartum deaths only comprised $6 \%$ of all stillbirths in our cohort. Our findings compare favourably with the reported mean intrapartum rates of $0.9 / 1000$ and $7 / 1000$ in developed and developing countries, respectively, during the mid2000s. ${ }^{10}$ Furthermore, approximately one third of our intrapartum stillbirths involved congenital lethal malformations; the patients had not undergone intrapartum fetal monitoring and no active 
resuscitation had been performed after birth. Our low intrapartum stillbirth rate was related to the use of continuous fetal heart rate monitoring, as well as short decision-to-delivery interval (median 10 minutes) and bradycardia-to-delivery interval (median 11 minutes); these approaches have been described in our previous reports. ${ }^{11-13}$

\section{Changes in stillbirth causes and risk factors}

In this study, we observed a significant decline in stillbirths caused by congenital malformations and chromosomal abnormalities since 2010. This coincided with implementation of the universal first trimester Down syndrome screening by the Hospital Authority in $2010,{ }^{14-16}$ as well as the provision of non-invasive cell-free fetal DNA testing in private sectors beginning in 2011.17,18 These two new services might have various implications beyond improvements in detection of fetuses with trisomy 21 . The early detection of lethal malformations and genetic disorders (eg, trisomy 18, trisomy 13 , and alpha-thalassemia major) was also improved. ${ }^{19}$ Because mothers were encouraged to undergo earlier antenatal assessments, fetuses with those lethal diseases were converted to legal abortions earlier during pregnancy; instead of progressing until stillbirth or neonatal death.

Pre-eclampsia is a known important causative factor of fetal death. ${ }^{20}$ In this study, pre-eclampsia was regarded as the leading cause of 24 stillbirths; eight of these cases (33.3\%) also had FGR that contributed to birthweight below the first centile. In addition, pre-eclampsia caused placental abruption in another 11 stillbirths; co-existing preeclampsia was present in one case of congenital abnormality (Haemoglobin Barts) and one case of chorioamnionitis. Therefore, the risk of stillbirth in the presence of pre-eclampsia was increased by six-fold, from $1.6 \%$ to $9.6 \%$ (Table 5 ). Although the prevalence of pre-eclampsia increased from $1.5 \%$ $(888 / 58$ 634) in $2000-09$ to $1.7 \%$ (1090/66 031) in 2010-19 (Table 6), the pre-eclampsia-related stillbirth rate decreased from $2.5 \%(22 / 888)$ to $1.4 \%$ $(15 / 1090)$. This reduction is probably related to improvements in antenatal care, early detection, and intervention (eg, iatrogenic preterm delivery). Because increased pre-eclampsia prevalence is related to advanced maternal age and obesity, ${ }^{7,21}$ primary prevention via first trimester screening and aspirin prophylaxis is essential for preventing adverse fetal outcomes in the future. ${ }^{22-25}$

We have noticed increasing rates of stillbirths related to placental pathologies, such as villitis or villous vasculopathy. Stillbirths were also associated with FGR; approximately $30 \%$ of cases involved birthweight below the third centile. Because placentas of livebirths were not subjected to routine histological examination, the prevalences of such pathologies remain unknown. Furthermore, these cases were categorised only according to postdelivery histology; the determinations were made after exclusion of obvious clinical abnormalities (eg, pre-eclampsia, medical diseases, congenital disorders, and genetic disorders). Therefore, such pathologies are challenging to diagnose prenatally with the goal of preventing fetal death, unless FGR is detected. Similarly, the 61 cases in the FGR group involved stillbirths without obvious causes of FGR. Their birthweight centiles were below first centile, first to below third centile, and third to less than fifth centile in $44(72.1 \%), 16(26.2 \%)$, and one (1.6\%) cases, respectively. However, a substantial proportion of these cases $(43.5 \%)$ were diagnosed based on post-delivery birthweight alone, rather than through prenatal assessments. Conventionally, fetal growth is routinely monitored at intervals of a few weeks by fundal height measurement in public sector hospitals. Only high-risk patients or suspected cases of FGR are monitored via sonographic fetal biometric measurement. Further service improvements should focus on increasing the rate of FGR detection, while minimising the falsepositive rate using a combination of first trimester biochemical markers. ${ }^{26-31}$

Unexplained stillbirths constituted $36.2 \%$ of our stillbirth cohort, which is consistent with the prevalences in other developed countries ${ }^{32}$; the prevalence in our study did not substantially decrease during the study period. With the exceptions of nulliparity and non-admitted status, we were unable to identify any maternal demographic factors that contributed to this type of stillbirth. Unexplained stillbirths were unrelated to extreme maternal age or extreme maternal BMI (ie, underweight or overweight). In $17.4 \%$ of these cases, the birthweight centile was between the third and less than tenth centile, which was much higher than expected. These might have constituted more subtle cases of FGR or late-onset FGR. Further studies are needed to determine whether late-onset FGR or elective induction of labour at 39 weeks can reduce the stillbirth rate in Hong Kong. Chorioamnionitis, placental abruption, and cord accidents are unpredictable events; their prevalences among stillbirths remained similar throughout the study period.

\section{Strengths and limitations}

To our knowledge, this is the largest study concerning the prevalence and causes of stillbirth in Hong Kong with a 20-year study period. The computerised database of the Hospital Authority provided comprehensive information concerning major risk factors, although it did not contain data concerning maternal social factors (eg, smoking, family income, and marital status). Clinical 
practice might have changed during the 20-year study period; this might have led to modifications regarding the classification of stillbirth causes. For example, the shift from karyotyping to chromosomal microarrays may facilitate more diagnoses of genetic diseases. ${ }^{33}$ Fetal growth restriction was classified based on birthweight centile; it might have been underestimated because fetal death might have occurred several days before birth. Thus, we used fetal weight below the third centile as a threshold to identify high-risk cases. Lastly, although our cohort provides extensive data regarding stillbirths in Hong Kong, the number of cases may have been insufficient to identify statistically significant results concerning less common events. Nonetheless, our findings provide a basis for future territory-wide reviews of perinatal outcomes. We will also investigate the neonatal mortality of singleton pregnancies and perinatal mortality of multiple pregnancies in our subsequent studies.

\section{Conclusion}

The overall perinatal mortality rate was significantly reduced from 2000-09 to 2010-19. We observed a declining trend in stillbirth rate among singleton pregnancies, mainly because of improvements in early prenatal diagnosis of congenital malformations and genetic disorders; this led to increasing termination of affected pregnancies before 24 weeks. The prevalence of pre-eclampsia has been rising because of the increasing maternal age, but the stillbirth rate among patients with pre-eclampsia has decreased. Fetal growth restriction of unknown cause contributes to $16 \%$ of all stillbirths; its prevalence has not changed over time. The majority of FGR cases were not diagnosed before birth. Overall, 36\% of stillbirths were unexplained, and they might have involved components of FGR. Therefore, prenatal FGR detection remains a priority for our obstetric service.

\section{Author contributions}

Concept or design: STK Wong, WT Tse, TY Leung. Acquisition of data: STK Wong, WT Tse, TY Leung. Analysis or interpretation of data: STK Wong, WT Tse, DS Sahota.

Drafting of the manuscript: STK Wong, SL Lau, TY Leung. Critical revision of the manuscript for important intellectual content: All authors.

All authors had full access to the data, contributed to the study, approved the final version for publication, and take responsibility for its accuracy and integrity.

\section{Conflicts of interest}

All authors have disclosed no conflicts of interest.

\section{Funding/support}

This research received no specific grant from any funding agency in the public, commercial, or not-for-profit sectors.

\section{Ethics approval}

Ethical approval was obtained from The Joint Chinese University of Hong Kong-New Territories East Cluster Clinical Research Ethics Committee (Ref CRE 2017.442).

\section{References}

1. Hong Kong College of Obstetricians \& Gynaecologists. Territory-wide Audit Report 2014. Available from: https:// www.hkcog.org.hk/hkcog/Download/Territory-wide_ Audit_in_Obstetrics_Gynaecology_2014.pdf. Accessed 11 Feb 2021.

2. Cousens S, Blencowe $\mathrm{H}$, Stanton $\mathrm{C}$, et al. National, regional, and worldwide estimates of stillbirth rates in 2009 with trends since 1995: a systematic analysis. Lancet 2011;377:1319-30.

3. World Health Organization. Maternal, newborn, child and adolescent health. Definition of stillbirths. Available from: https://www.who.int/maternal_child_adolescent/ epidemiology/stillbirth/en/ Accessed 11 Feb 2021.

4. Blencowe H, Cousens S, Jassir FB, et al. National, regional, and worldwide estimates of stillbirth rates in 2015, with trends from 2000: a systematic analysis. Lancet Glob Health 2016;4:e98-108.

5. Lawn JE, Blencowe $H$, Pattinson $R$, et al. Stillbirths: Where? When? Why? How to make the data count? Lancet 2011;377:1448-63.

6. de Bernis L, Kinney MV, Stones W, et al. Stillbirths: ending preventable deaths by 2030. Lancet 2016;387:703-16.

7. Leung TY, Leung TN, Sahota DS, et al. Trends in maternal obesity and associated risks of adverse pregnancy outcomes in a population of Chinese women. BJOG 2008;115:152937.

8. Chan SS, Lau AP, To KF, Leung TY, Lau TK, Leung TN. Umbilical cord ulceration as a cause of fetal haemorrhage and stillbirth. Hong Kong Med J 2008;14:148-51.

9. Lau TK, Li CY. A perinatal audit of stillbirths in a teaching hospital in Hong Kong. Aust N Z J Obstet Gynaecol 1994;34:416-21.

10. Goldenberg RL, McClure EM, Bann CM. The relationship of intrapartum and antepartum stillbirth rates to measures of obstetric care in developed and developing countries. Acta Obstet Gynecol Scand 2007;86:1303-9.

11. Leung TY, Chung PW, Rogers MS, Sahota DS, Lao TT, Chung TK. Urgent Cesarean delivery for fetal bradycardia. Obstet Gynecol 2009;114:1023-8.

12. Leung TY, Lao TT. Timing of Caesarean section according to urgency. Best Pract Res Clin Obstet Gynaecol 2013;27:251-67.

13. Wong L, Tse WT, Lai CY, et al. Bradycardia-to-delivery interval and fetal outcomes in umbilical cord prolapse. Acta Obstet Gynecol Scand 2021;100:170-7.

14. Sahota DS, Leung WC, Chan WP, To WW, Lau ET, Leung TY. Prospective assessment of the Hong Kong Hospital Authority universal Down syndrome screening programme. Hong Kong Med J 2013;19:101-8.

15. Sahota DS, Leung TY, Fung TY, Chan LW, Law LW, Lau TK. Medians and correction of biochemical and ultrasound markers in Chinese undergoing first trimester screening for Trisomy 21. Ultrasound Obstet Gynecol 2009;33:38793.

16. Leung TY, Chan LW, Leung TN, et al. First-trimester 
combined screening for trisomy 21 in a predominantly Chinese population. Ultrasound Obstet Gynecol 2007;29:14-7.

17. Cheng Y, Leung WC, Leung TY, et al. Women's preference for non-invasive prenatal DNA testing versus chromosomal microarray after screening for Down syndrome: a prospective study. BJOG 2018;125:451-9.

18. Chan YM, Leung WC, Chan WP, Leung TY, Cheng YK, Sahota DS. Women's uptake of non-invasive DNA testing following a high-risk screening test for trisomy 21 within a publicly funded healthcare system: findings from a retrospective review. Prenat Diagn 2015;35:342-7.

19. Leung TY, Vogel I, Lau TK, et al. Identification of submicroscopic chromosomal aberrations in fetuses with increased nuchal translucency and an apparently normal karyotype. Ultrasound Obstet Gynecol 2011;38:314-9.

20. Harmon QE, Huang L, Umbach DM, et al. Risk of fetal death with preeclampsia. Obstet Gynecol 2015;125:628-35.

21. Chaemsaithong P, Leung TY, Sahota D, et al. Body mass index at 11-13 weeks' gestation and pregnancy complications in a Southern Chinese population: a retrospective cohort study. J Matern Fetal Neonatal Med 2019;32:2056-68.

22. Chaemsaithong P, Sahota D, Pooh RK, et al. First-trimester pre-eclampsia biomarker profiles in Asian population: a multicenter cohort study. Ultrasound Obstet Gynecol 2020;56:206-14.

23. Chaemsaithong P, Pooh RK, Zheng M, et al. Prospective evaluation of screening performance of first-trimester prediction models for preterm preeclampsia in Asian population. Am J Obstet Gynecol 2019;221:650.e1-16.

24. Cheng YK, Poon LC, Shennan A, Leung TY, Sahota DS. Inter-manufacturer comparison of automated immunoassays for the measurement of soluble FMS-like tyrosine kinase-1 and placental growth factor. Pregnancy Hypertens 2019;17:165-71.
25. Cheng Y, Leung TY, Law LW, Ting YH, Law KM, Sahota DS. First trimester screening for pre-eclampsia in Chinese pregnancies: case-control study. BJOG 2018;125:442-9.

26. Li W, Chung CY, Wang CC, et al. Monochorionic twins with selective fetal growth restriction: insight from placental whole transcriptome analysis. Am J Obstet Gynecol 2020;223:749.e1-16.

27. Meng M, Cheng YK, Wu L, et al. Whole genome miRNA profiling revealed miR-199a as potential placental pathogenesis of selective fetal growth restriction in monochorionic twin pregnancies. Placenta 2020;92:44-53.

28. Cheng YK, Lu J, Leung TY, Chan YM, Sahota DS. Prospective assessment of the INTERGROWTH-21 and World Health Organization estimated fetal weight reference curve. Ultrasound Obstet Gynecol 2018;51:7928.

29. Cheng Y, Leung TY, Lao T, Chan YM, Sahota DS. Impact of replacing Chinese ethnicity-specific fetal biometry charts with the INTERGROWTH-21(st) standard. BJOG 2016;123 Suppl 3:48-55.

30. Leung TY, Chan LW, Leung TN, Fung TY, Sahota DS, Lau TK. First trimester maternal serum levels of placental hormones are independent predictors of second trimester fetal growth parameters. Ultrasound Obstet Gynecol 2006;27:156-61.

31. Leung TY, Sahota DS, Chan LW, et al. Prediction of birth weight by crown-rump length and maternal serum levels of pregnancy-associated plasma protein-A in the first trimester. Ultrasound Obstet Gynecol 2008;31:10-4.

32. Reinebrant HE, Leisher SH, Coory $M$, et al. Making stillbirths visible: a systematic review of globally reported causes of stillbirth. BJOG 2018;125:212-24.

33. Hui AS, Chau MH, Chan YM, et al. The role of chromosomal microarray analysis among fetuses with normal karyotype and single system anomaly or nonspecific sonographic findings. Acta Obstet Gynecol Scand 2021;100:235-43. 